\title{
Brain Activity Associated with Expectancy-Enhanced Placebo Analgesia as Measured by Functional Magnetic Resonance Imaging
}

\author{
Jian Kong, ${ }^{1}$ Randy L. Gollub, ${ }^{1,2,3}$ Ilana S. Rosman, ${ }^{1}$ J. Megan Webb, ${ }^{1}$ Mark G. Vangel, ${ }^{2,3}$ Irving Kirsch, ${ }^{4}$ and \\ Ted J. Kaptchuk ${ }^{5}$ \\ ${ }^{1}$ Department of Psychiatry, Massachusetts General Hospital (MGH), ${ }^{2}$ MGH/Massachusetts Institute of Technology/Harvard Medical School (HMS) \\ Athinoula A. Martinos Center for Biomedical Imaging, and ${ }^{3} \mathrm{MGH}$ General Clinical Research Center Biomedical Imaging Core, Charlestown, Massachusetts \\ 02129, ${ }^{4}$ School of Applied Psychosocial Studies, University of Plymouth, Plymouth, Devon, PL4 8AA, United Kingdom, and ${ }^{5}$ Osher Institute, HMS, Boston, \\ Massachusetts 02215
}

In this study, a well established expectancy manipulation model was combined with a novel placebo intervention, a validated sham acupuncture needle, to investigate the brain network involved in placebo analgesia. Sixteen subjects completed the experiment. We found that after placebo acupuncture treatment, subjective pain rating reduction (pre minus post) on the placebo-treated side was significantly greater than on the control side. When we calculated the contrast that subtracts the functional magnetic resonance imaging (fMRI) signal difference between post-treatment and pretreatment during pain application on placebo side from the same difference on control side [e.g., placebo (post - pre) - control (post - pre)], significant differences were observed in the bilateral rostral anterior cingulate cortex (rACC), lateral prefrontal cortex, right anterior insula, supramarginal gyrus, and left inferior parietal lobule. The simple regression (correlation) analysis between each subject's fMRI signal difference of post-treatment and pretreatment difference on placebo and control side and the corresponding subjective pain rating difference showed that significant negative correlation was observed in the bilateral lateral/orbital prefrontal cortex, rACC, cerebellum, right fusiform, parahippocampus, and pons. These results are different from a previous study that found decreased activity in pain-sensitive regions such as the thalamus, insula, and ACC when comparing the response to noxious stimuli applied to control and placebo cream-treated areas of the skin. Our results suggest that placebo analgesia may be configured through multiple brain pathways and mechanisms.

Key words: placebo; placebo analgesia; expectancy; expectancy manipulation; pain; placebo acupuncture needle; Steitberger needle

\section{Introduction}

Placebo analgesia is the one of the most robust and best-studied placebo effects (Hoffman et al., 2005). The neurobiology of placebo, an understanding of the physiological substrate beyond psychological theories, was born with the discovery that naloxone can reverse placebo analgesia (Levine et al., 1978; Amanzio and Benedetti, 1999; Benedetti et al., 1999). Other studies indicate that endogenous opioids alone cannot account for placebo analgesia and the process may be configured through different mechanisms. For instance, experimental reports have shown that placebo analgesia may occur without the involvement of endogenous opioids or that under certain circumstance, naloxone only partially blocks the placebo analgesia effect (Gracely et al., 1983; Grevert et al., 1983; Amanzio and Benedetti, 1999).

The recent advent of brain imaging tools provides scientists

Received Aug. 22, 2005; revised Nov. 10, 2005; accepted Nov. 11, 2005.

This work was supported by a Harvard Medical School Osher pilot grant to J.K., National Institutes of Health (NIH) Grants R21 AT00949 and P01-AT002048 to R.L.G., and NIH Grant R21 AT002860 to T.J.K.

Correspondence should be addressed to Jian Kong, 14913th Street, Room 2661, Charlestown, MA 02129. E-mail: kongj@nmr.mgh.harvard.edu.

D0I:10.1523/JNEUROSCI.3556-05.2006

Copyright $\odot 2006$ Society for Neuroscience $\quad$ 0270-6474/06/260381-08\$15.00/0 with a window into the brain activity orchestrating the effect of placebo analgesia (Petrovic et al., 2002; Lieberman et al., 2004; Wager et al., 2004; Zubieta et al., 2005). Petrovic et al. (2002) found that both opioid analgesia and placebo analgesia, but not the pain-only condition, led to increased cerebral blood flow in brain regions known to be rich in opioid receptors such as the rostral anterior cingulate cortex (rACC) and the periaqueduct gray. Wager et al. (2004) investigated functional magnetic resonance imaging (fMRI) signal changes during the anticipation and experience of pain and found decreased activity in pain-sensitive regions such as the thalamus, insula, and ACC when comparing the response to noxious stimuli applied to control and placebo cream-treated areas of the skin. These studies provide evidence for potential neural mechanisms involved in placebo analgesia. However, one interesting question that remains to be answered is whether these observations of an analgesic effect can be replicated when using a placebo vehicle that mimics a culturally novel medical therapy.

In this experiment, we combined a well established expectancy manipulation that has been shown to reliably enhance the placebo response (Voudouris et al., 1990; Montgomery and Kirsch, 1997; Price et al., 1999) and a validated sham acupuncture 
device (Streitberger and Kleinhenz, 1998; Kleinhenz et al., 1999; White et al., 2003; Kong et al., 2005a) to investigate the fMRI signal changes during pain administration before and after placebo acupuncture treatment. Recently, a positron emission tomography study by Pariente et al. (2005) in osteoarthritis patients showed that treatment (stimulation) with real acupuncture (RA), placebo acupuncture with Steitberger needle (SN), and overt placebo (OP) evoked different brain activation patterns, but this study did not establish an analgesic effect from either acupuncture or placebo treatment.

Investigating the mechanism of analgesic effect evoked by placebo acupuncture has potential for advancing our understanding of placebo phenomenon. Unlike pills, ointments, and injections, acupuncture is novel and unfamiliar to many individuals in Western culture. Because acupuncture is embedded in unique beliefs and rituals, it may have distinct mechanisms from other treatment modalities (Kaptchuk, 2002b). Also, a lack of understanding the relationship between acupuncture and placebo has become a barrier to advancing research in this field (Kaptchuk, 2002a).

\section{Materials and Methods \\ Subjects}

Twenty-four right-handed healthy subjects (13 males; mean age, 28.4 years $\pm 6.6 \mathrm{SD}$ ) who were naive to acupuncture participated in this study. Experiments were conducted with the understanding and written consent of each subject and approval by the Human Subjects Committee at Massachusetts General Hospital. At the end of the experiment, all the subjects were told about the true nature of the experiment, because they had been recruited with the understanding that this was a study about acupuncture analgesia.

\section{Procedures for the delivery and assessment of noxious} thermal stimuli

All subjects were recruited to participate in two behavioral testing sessions and one fMRI scanning session (supplemental Fig. 1, available at www.jneurosci.org as supplemental material). Each session was separated by a minimum of $4 \mathrm{~d}$.

Calibrated thermal pain stimuli were delivered to the right medial aspect of the forearm using a TSA-2001 Thermal Sensory Analyzer with a $3 \times 3 \mathrm{~cm}$ probe (Medoc Advanced Medical Systems, Rimat Yishai, Israel) running proprietary computerized visual analog scale software (CoVAS) (Davis et al., 1998; Peyron et al., 1999; Kwan et al., 2000). All stimuli were initiated from a baseline resting temperature of $32^{\circ} \mathrm{C}$ and increased to a target temperature. Each stimulus was presented for $10 \mathrm{~s}$, including $2.5 \mathrm{~s}$ each for ramp up and down. The minimum interstimulus interval was $16 \mathrm{~s}$. To improve the consistency of the pain stimuli and to ensure that the same area of skin was tested before and after treatment in sessions 2 and 3 , we used a marker to draw a numbered, two-by-three grid on the medial aspect (palmar side) of the right forearm so that there were three boxes on the radial side and three boxes on the ulnar side (Fig. 1, diagram). We placed the thermal probe in one box of the grid for each of the stimuli sequences [e.g., random sequence (RS) and identical sequence (IS)]. For details, see Figure 1 and the following.

Session 1. We used the first behavioral session to determine appropriate stimulus intensities for each subject, to minimize anticipatory anxi-
Detailed experimental procedure

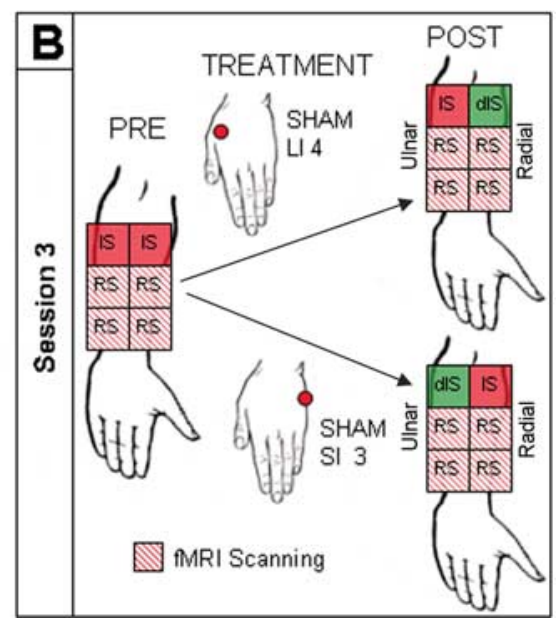
Figure 1. Details of experimental procedure. $\boldsymbol{A}$, In session 2, we used a marker to draw a numbered two-by-three grid on the
medial aspect of the right forearm. We placed the thermal probe in one box of the grid for each of the stimulus sequences (e.g., RS pretreatment and post-treatment difference in pain rating and fMRI signal change during the four RSs applied on placebo and control sides are the primary outcomes of this study.

ety, to control for rating strategy and learning effects, and to teach the subjects to rate the stimuli using the Sensory Box and Affective Box 0-20 scales (Gracely et al., 1978a,b, 1979). This session consisted of two phases: ascending series and random sequence.

Subjects first experienced an ascending series of calibrated heat stimuli (the first stimulus was increased from the baseline of $32^{\circ} \mathrm{C}$ to a target of $38^{\circ} \mathrm{C}$ and the target temperatures for the following stimuli increased by $1{ }^{\circ} \mathrm{C}$ to the subjects' tolerance or to a maximum of $52^{\circ} \mathrm{C}$ ) on both arms. Temperatures that elicited subjective intensity ratings in the low pain range $(8-11$; the mild to moderate range on the $0-20$ Sensory Box scale) and high pain range (14-17; the strong to intense range on the $0-20$ Sensory Box scale) were selected for each subject.

Then, we applied an RS to the right arm. In the RS, two intensity levels of stimuli (high and low) were each presented an equal number of times in a random order. Temperatures were adjusted when necessary to ensure that subjective ratings of the temperature established for that subject as high or low were in the desired range. By the end of this session, high and low intensities (temperatures) were determined, and these temperatures were used in the subsequent sessions. The RS provided a way to determine how reliably a subject could rate noxious stimuli of different intensities when they were presented in an unpredictable order. Another advantage of using two levels of stimuli intensity in this random sequence is that when analyzing the fMRI data, the fMRI contrast between these two levels enabled us to easily locate the brain regions that have pain intensity correlated activation.

Session 2. The second behavioral session (Fig. 1A) was designed to detect whether subjects could reliably rate calibrated noxious heat stimuli and to manipulate the subjects' expectancy of acupuncture analgesia. The session consisted of four phases: application of RS, pretreatment IS, placebo acupuncture, and post-treatment expectancy manipulation, which consisted of the repeated application of IS and RS with lowered temperatures.

At the beginning of the session, we told the subjects that this was a study about acupuncture analgesia. Then, each subject read an introduction to acupuncture analgesia that explained that although acupuncture has been used for 2000 years, the mechanism behind its analgesia effects remains unclear. Furthermore, the introduction stated that some people 
have a very positive response to acupuncture treatment and are called "good acupuncture responders." On the other hand, some people do not respond well to acupuncture and are called "poor acupuncture responders." And finally, it stated that once a person is established as a good or poor responder to acupuncture, that person will always remain a good or poor responder.

After the subjects read the introduction, we displayed a figure depicting how the acupuncture meridian lines connect a set of acupuncture points according to Traditional Chinese Medicine. For this study, we used either the large intestine (LI) meridian, which runs along the radial side of the hand and forearm, or the small intestine (SI) meridian, which runs along the ulnar side of the hand and forearm. Then, we told the subjects that according to acupuncture theory and previous reports, acupuncture would only produce an analgesic effect on the side of the arm where needles were placed (placebo side) but not on the other side of the arm where there were no needles (control side). From this information, subjects would expect a needle on the thumb side of the hand (LI) to have an effect along the radial side of the palmar forearm. Conversely, they would expect a needle on the pinky (fifth metacarpal) side of the hand (SI) to have an effect along the ulnar side of the palmar forearm. Finally, the subjects were told that if they are good responders, they would feel a difference between the treated (placebo) and untreated (control) sides of the arm after the acupuncture treatment. In fact, the above information may not be true.

In the RS, three high and three low stimuli were presented on four of the six boxes, as drawn on the medial forearm (Fig. $1 \mathrm{~A}$, PRE). The presentation of these four random sequences alternated between the radial and ulnar sides of the medial surface of the forearm. Subject ratings of high and low temperatures were evaluated for consistency. Only subjects whose subjective pain ratings clearly corresponded to the magnitude of the stimulus intensity (i.e., the average rating of high pain stimuli was higher than that of low pain stimuli in each RS) continued in the study.

In pretreatment IS, we applied six stimuli set at an intensity level halfway between high and low (Fig. 1). For example, a subject who received $47^{\circ} \mathrm{C}$ for low and $50^{\circ} \mathrm{C}$ for high stimuli would have a stimulus temperature in the IS of $48.5^{\circ} \mathrm{C}$. The consistency in the IS enabled us to better manipulate subjects' expectancy of acupuncture analgesia by providing a way for subjects to easily compare stimulus intensity before and after treatment.

Then, we administered a placebo acupuncture treatment to one side of the arm. Subjects were randomized into two groups to receive placebo acupuncture at one of two nonacupuncture points, either sham large intestine 4 (LI 4) or sham small intestine 3 (SI 3) (Fig. $1 A$ ). Sham LI 4 was located at the midpoint of the first metacarpal $\sim 1 \mathrm{~cm}$ lateral to the true point on the hand. Sham SI 3 was located at the midpoint of the true SI 3 and SI 4 on the hand. In this way, either the radial (LI) or ulnar (SI) side of the forearm became the placebo or control side. The treatment lasted $\sim 5$ min. To administer the treatment, we used a placebo acupuncture needle inserted through a small, tape-covered plastic ring. This validated sham device (Streitberger and Kleinhenz, 1998; Kleinhenz et al., 1999; White et al., 2003; Kong et al., 2005a) retracts into its casing when pressed on the skin, similar to the action of a retractable stage knife. After placement, the needle was briefly twirled to simulate acupuncture manipulation and then left in place for $5 \mathrm{~min}$ before being withdrawn.

After the placebo acupuncture treatment, we told the subjects that we would administer the same stimuli sequences as before the treatment to test the analgesic effect of acupuncture except that this time we would begin with the IS instead of the RS. To manipulate the subjects' expectancy of the analgesic effect, we surreptitiously decreased the temperature of all noxious stimuli by $4-6^{\circ} \mathrm{C}$ on whichever side of the arm had received treatment (placebo side) but used the same stimuli temperature as before on the nontreated side (control side). Such a reduction on the placebo side should elicit a rating of "faint to weak" (1-5 on the Gracely Sensory Box scale) and was designed to give subjects an unmistakable experience of profound analgesia in both identical and random stimuli sequences. We began with the decreased identical sequence (dIS) because we wanted to make the perception of analgesia as obvious as possible, reasoning that it would be easier to detect a temperature reduction across the arm in a sequence of identical stimuli than in the decreased random sequence (dRS) presentation of high and low stimuli (Fig. 1 A, POST). This procedure mirrored an expectancy manipulation model in which some areas of the arm receive a placebo analgesia cream while adjacent areas act as a control (Voudouris et al., 1990; Montgomery and Kirsch, 1997; Price et al., 1999; De Pascalis et al., 2002; Wager et al., 2004).

Each subject completed an expectation scale indicating how much pain relief they expected from acupuncture treatment, with 0 indicating a very negative expectation of pain relief and 20 indicating a very positive expectation of pain relief. Each subject completed the scale twice, once before placebo acupuncture treatment and once after the expectancy manipulation procedure.

Session 3. Session 3 was performed in the fMRI scanner (Fig. 1B). Subjects were told we would repeat the procedures of session 2 during scanning to investigate the brain network involved in acupuncture analgesia.

In this session, we first administered the same pretreatment stimuli sequences, two RSs and one IS on placebo and control sides for a total of six stimulus sequences as in session 2, except the RS consisted of six high pain and six low pain stimuli for 12 total instead of six total stimuli. Then, subjects received an identical sham acupuncture treatment on the same side (placebo side) as they did in session 2 (Fig. $1 B$, PRE).

After treatment, subjects were told that we would again present the same stimuli sequences to test the acupuncture analgesia effect. As we did in session 2, we began with the identical sequence using the decreased stimuli temperature (dIS) on the placebo side and the original temperature on control side (IS). We intended for this surreptitious reduction to serve as a supplemental prompt to "remind" subjects of the analgesia they experienced after the previous treatment in session 2 .

Then, the four RSs were delivered at the original stimulus temperature on both placebo and control sides to test for an analgesia effect evoked by placebo treatment (Fig. 1B, POST). The differences between placebo and control sides on pain rating and brain activation during these four random sequences were the primary outcomes of this study.

Before the acupuncture treatment and after experiencing the dIS on the placebo side and the IS on the control side, subjects were required to complete the expectation scale, again indicating how much pain relief they expected from acupuncture treatment.

fMRI scanning was performed only during the administration of the full-strength RSs before and after placebo treatment. During scanning, subjects were instructed to keep their eyes open and fixed on a small black fixation cross in the center of a screen in front of them. The cross turned red to cue the subject to the onset and duration of each stimulus (10 s). Each stimulus was followed by a fixed delay of $4 \mathrm{~s}$, at the start of which the cross turned black again. Then, we displayed the Sensory Box scale on the screen for $10 \mathrm{~s}$. Subjects were instructed to use two buttons to move a pointer up or down to indicate their subjective rating of the preceding stimulus. The next stimulus began after a variable time delay of 2-12 s (average, $6 \mathrm{~s}$ ). Before scanning, all subjects were required to practice using the key press until they were confident in their ability to perform all aspects of the rating task.

\section{fMRI data acquisition and analysis}

All brain imaging was performed with a three-axis gradient head coil in a 3 tesla head only Siemens AG (Erlangen, Germany) MRI System equipped for echo planar imaging. Thirty axial slices ( $4 \mathrm{~mm}$ thick with 1 $\mathrm{mm}$ skip) parallel to the anterior and posterior commissure covering the entire brain were imaged with $2000 \mathrm{~ms}$ repetition time, $40 \mathrm{~ms}$ echo time, $90^{\circ}$ flip angle, and $3.13 \times 3.13 \mathrm{~mm}$ in-plane spatial resolution. A highresolution three-dimensional magnetization-prepared rapid-acquisition gradient echo sequence was also collected for anatomic localization.

Preprocessing and statistical analysis were performed using SPM2 software (Wellcome Department of Cognitive Neurology, London, UK). Preprocessing included motion correction, normalization to the Montreal Neurological Institute (MNI) stereotactic space, and spatial smoothing with an $8 \mathrm{~mm}$ Gaussian kernel. Then, for each subject, the eight contrasts (placebo and control side-by-pretreatment and posttreatment-by-high pain and low pain) with the fixation (baseline) were calculated with a general linear model. To prevent spurious deactivations, global signal scaling was not applied. Low-frequency noise was 
Table 1. Subjective pain ratings of identical pain stimuli before and during expectancy manipulation on placebo side (IS, dIS) and control side (IS, IS) in sessions 2 and $3(n=16$; mean \pm SD)

\begin{tabular}{llrlll}
\hline & \multicolumn{2}{l}{ Session 2 } & & \multicolumn{2}{l}{ Session 3 } \\
\cline { 2 - 3 } \cline { 5 - 6 } & Before & During & & Before & \multicolumn{1}{c}{ During } \\
\hline Placebo side & $12.9 \pm 2.3$ & $3.5 \pm 2.1$ & & $12.4 \pm 2.5$ & $2.5 \pm 2.3$ \\
Control side & $13.2 \pm 1.8$ & $12.8 \pm 2.4$ & & $11.9 \pm 3.2$ & $12.4 \pm 3$ \\
\hline
\end{tabular}

removed with a high-pass filter applied with default values to the fMRI time series at each voxel.

Group analysis was performed using a random-effects model. A oneway ANOVA (within-subject) was performed across all subjects who finished the study. Nonsphericity correction was performed, the replication was over subjects, and correlated repeated measures were chosen. Then, we compared all pretreatment high pain with low pain on both placebo and control sides, as was done in a previous study (Wager et al., 2004), to generate a mask for brain regions that have pain stimulus intensity correlated activations within which to test for the placebo effect. According to previous imaging studies on placebo analgesia and pain modulation (Petrovic et al., 2002; Lieberman et al., 2004; Wager et al., 2004), brain regions such as the ACC, dorsal lateral prefrontal cortex (DLPFC), and orbital prefrontal cortex play an important role in pain modulation, so we added them to our a priori regions of interest (ROIs).

To investigate the brain network involved in placebo analgesia, we calculated the difference of post-treatment minus pretreatment differences in response to application of the RS stimuli on both the placebo side and control side (e.g., placebo increases relative to control [placebo (post - pre) - control (post - pre)] and placebo decreases relative to control [control (post - pre) - placebo (post - pre)]).

The threshold of activation within the mask (pretreatment high pain $>$ low pain) and the predefined ROIs (ACC, DLPFC, and orbital prefrontal cortex) was set at voxel-wise $p<0.005$ uncorrected with 20 contiguous voxels. A threshold of voxel-wise $p<0.001$ uncorrected with 20 contiguous voxels and cluster $p<0.05$ corrected was used for activation in other regions.

In another second-level analysis, a simple regression (correlation) analysis was calculated between each subject's fMRI signal difference of pretreatment and post-treatment difference on placebo and control sides in response to RS pain application [e.g., placebo (post - pre) - control (post - pre)] and the corresponding subjective pain rating changes. The threshold was set to voxel-wise $p<0.005$ uncorrected with 20 contiguous voxels.

\section{Results \\ Subjects}

Of the 24 volunteers who consented to participate in the study, 16 (nine male) completed all sessions. Five subjects could not reliably distinguish the high pain intensity stimuli from low pain intensity stimuli in session 2 and were dropped from the study. Three subjects were dropped because of incomplete fMRI data sets.

\section{Subjective ratings of pain and expectancy}

Table 1 shows that during manipulation in both sessions 2 and 3, subjects reported the expected large decrease in subjective pain rating in response to the surreptitiously lowered stimuli (dIS) applied to the placebo side but not on the control side in response to repeated IS. Note that before manipulation, ratings of IS are comparable on the two sides (Pre- vs Pre-).

After the expectancy manipulation in session 2, every subject gave a higher rating of expected pain relief from acupuncture with mean ratings on the $0-20$ expectation scale increasing from $12.6 \pm 3.3$ before manipulation to $18.4 \pm 1.5$ after manipulation $(p<0.000)$. In session 3 , their expectation of pain relief was maintained at a higher level, with a slight but significant decrease $(p<0.002)$ from session 2 before manipulation, but there was no difference after manipulation $(17.3 \pm 2.4$ before and $18.1 \pm 1.5$ after).

The pretreatment and post-treatment subjective ratings of the RS on both placebo and control sides in session 3 are shown in Table 2. The ANOVA of pretreatment rating (control and placebo, high pain and low pain) shows that the high pain rating is significantly greater than the low pain rating $(p<0.0001)$. There was no significant difference between the two sides or any interaction with pain level. We used the pretreatment and posttreatment difference in subjective pain ratings of the RS between placebo and control sides to detect placebo analgesia. The ANOVA on pain rating differences (pre minus post) between placebo and control side across two pain stimuli levels (control and placebo, high pain and low pain) showed that subjective pain rating reduction on the placebo side was significantly greater than it was on the control side $(p<0.0001)$ after treatment. There was no interaction between high pain and low pain $(p<0.26)$ on pretreatment minus post-treatment rating differences. Thus, we used all the pain stimuli in the following fMRI analysis.

\section{fMRI results}

To elucidate the brain regions that correlate with the intensity of pain stimulation, we calculated a contrast between all pretreatment high pain and low pain (high pain $>$ low pain). The comparison yielded significant activations (voxel-wise $p<0.005$ uncorrected with 20 contiguous voxels) in the entire predicted network of pain-sensitive regions including bilateral insular/ opercular cortices, ACC/medial prefrontal cortex (MPFC), SII, thalamus, cerebellum, brainstem, and left SI/M1 (contralateral) corresponding to the arm (Fig. $2 \mathrm{~A}$ ). The result from this contrast was used as a mask for the following analysis.

When we calculated the contrast that subtracts the fMRI signal difference between post-treatment and pretreatment during pain application on placebo side from the same difference on control side [e.g., placebo (post - pre) - control (post - pre)], the right anterior insula (AI) was the only region that showed significantly greater fMRI signal changes within the mask (Fig. $2 B$ ). Outside the mask, significant differences were also observed in bilateral lateral prefrontal cortex, rACC, left inferior parietal lobule, and right supramarginal gyrus. The detailed results are shown in Table 3.

Figure 2, $B$ and $C$, shows the location and graphs the estimated $\beta$ values of the peak activation voxel during application of RS pain stimuli in each of the eight conditions (placebo and control side-by-pretreatment and post-treatment-by-high pain and low pain) for the right AI within the mask and the bilateral rACC outside the mask. The right AI was activated by both high and low pain; this activation was temperature (intensity) dependent because the high pain evoked greater activation than low pain on both sides as well as before and after treatment. After placebo acupuncture treatment, the magnitude of activation was unchanged on the control side, whereas on the placebo side, the response was enhanced for both pain levels. Bilateral rACC was not active during pretreatment RS pain application on either side or after treatment on the control side. This region was uniquely activated during the RS stimulation after the placebo treatment on the placebo side.

When we calculated the contrast that subtracts the fMRI signal difference between post-treatment and pretreatment during pain application on the control side from the same difference on the placebo side [e.g., control (post - pre) - placebo (post - 
Table 2. Average pain sensory rating of random sequence stimuli on both placebo and control sides across two pain levels in session 3 ( $n=16 ;$ mean \pm SD)

\begin{tabular}{|c|c|c|c|c|c|c|}
\hline & \multicolumn{3}{|l|}{ Low pain } & \multicolumn{3}{|l|}{ High pain } \\
\hline & Before treatment & After treatment & Difference & Before treatment & After treatment & Difference \\
\hline Placebo side & $9.4 \pm 3.9$ & $8.8 \pm 3.7$ & $0.6 \pm 1.2^{*}$ & $13.5 \pm 2.1$ & $12.9 \pm 2.7$ & $0.6 \pm 1.4^{*}$ \\
\hline Control side & $8.9 \pm 3.6$ & $9.2 \pm 3.6$ & $-0.3 \pm 0.6^{*}$ & $13.6 \pm 2.0$ & $14.3 \pm 2.1$ & $-1.0 \pm 1.1^{*}$ \\
\hline
\end{tabular}

*Significant difference $(p<0.0001)$.

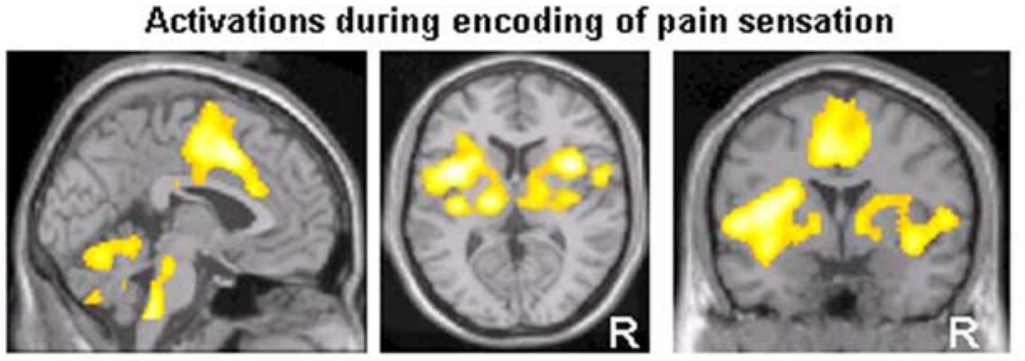

Representative regions involved in placebo analgesia
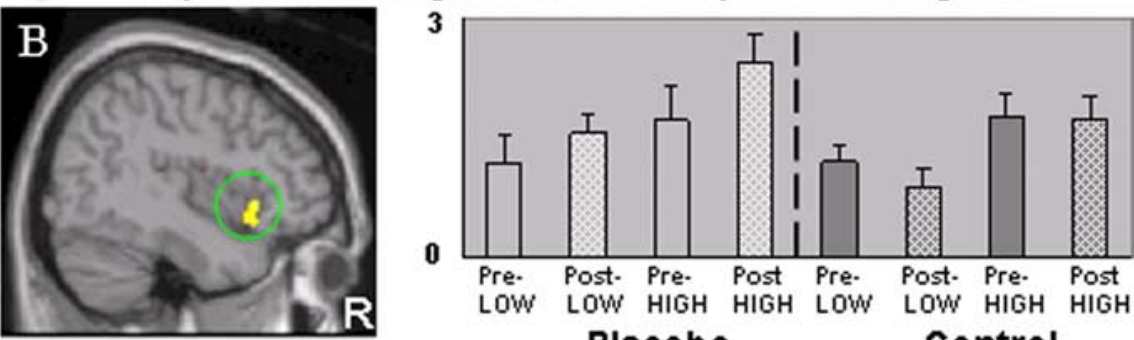

Placebo
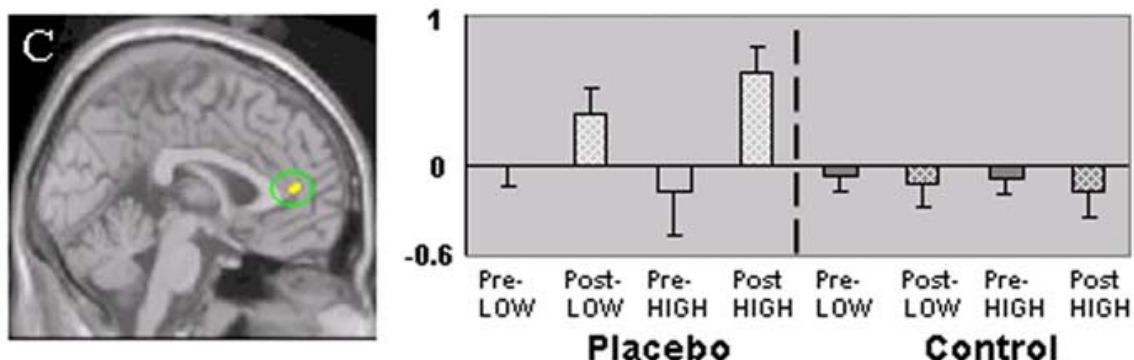

Figure 2. $\quad \boldsymbol{A}, \mathrm{fMRI}$ signal changes evoked by all pretreatment high pain minus all pretreatment low pain stimuli applied on both placebo and control side. Brain regions including the bilateral insular/opercular cortices, ACC/MPFC, cerebellum, and brainstem showed activation above threshold ( $p<0.005$; uncorrected with 20 contiguous voxels). These regions were used as a mask for the following fMRI analysis. $\boldsymbol{B}, \boldsymbol{C}$, Representative regions revealed by the contrast of the post-treatment and pretreatment difference (post minus pre) on the control side subtracted from the same difference on the placebo side [e.g., placebo (post pre) - control (post - pre)]. B shows activation in right anterior insula $(46,20,-4)$, and $C$ shows activation in bilateral rACC (2, 44,10 ). The bar graphs show the fMRI contrast estimated $\beta$ value at peak activation on both sides for both pain levels (mean \pm SE). Pre-LOW, Pretreatment low pain; Pre-HIGH, pretreatment high pain; Post-LOW, post-treatment low pain; Post-HIGH, posttreatment high pain; $R$, right side.

pre)], left M1 was the only region that showed a significant difference in level of activation (Table 3 ).

The result of simple regression (correlation) analysis between each subject's fMRI signal difference of pretreatment and posttreatment difference on placebo and control side in response to RS pain application [e.g., placebo (post - pre) - control (post pre)] and the corresponding pain rating difference is shown in Table 4. Significant negative correlations were observed in the bilateral lateral/orbital prefrontal cortex, rACC, and cerebellum, right fusiform, parahippocampus and pons, which means that the stronger the analgesia effect, the greater the activity in these regions. No region showed significant positive correlation.

\section{Discussion}

In this study, we combined an expectancy manipulation and placebo acupuncture treatment to investigate the brain regions involved in expectancy-induced analgesia. One difficulty in studying placebo analgesia using healthy volunteers and experimentally induced pain is that the placebo effects seem to be fewer and lower than those in genuine clinical pain studies (Beecher, 1959; Roberts et al., 1993). We found that after enhancement of expectancy to acupuncture analgesia, subjective pain rating reduction (pre minus post) on the placebo side was significantly greater than on the control side. This suggests that the culturally novel intervention of acupuncture can produce detectable placebo analgesia after expectancy manipulation. These methods can be applied to future studies investigating the mechanism underlying acupuncture analgesia, placebo analgesia, and their interrelationship.

In recent years, the AI has been noted for its possible role in transforming pain sensation to cognition (Craig et al., 2000; Craig, 2002, 2003; Kong et al., 2005b). One suggestion is that as interoceptive input moves from sensation to cognition, sensory representation in the posterior insula is initially rerepresented in the ipsilateral $\mathrm{AI}$ and then remapped to the right $\mathrm{AI}$ by the corticocortical and/or callosal pathways. The second-order rerepresentation in the right $\mathrm{AI}$ is proposed to subserve subjective feelings (Craig et al., 2000; Craig, 2002, 2003), which makes it an important candidate region for cognitive modulation of pain perception. Studies by Critchley and colleagues highlight the important role of the right AI in the conscious experiencing of bodily arousal and representation of these states as subjective feelings (Critchley et al., 2002, 2004). In this study, we found that after placebo treatment, fMRI signal in the right AI was enhanced for both pain levels on the placebo side; no effect of treatment was observed on the control side (Fig. $2 B$ ). This suggests that the AI has an important role during placebo modulation of pain perception.

In this study, we observed increased activation in $\mathrm{rACC}$ when comparing differences in fMRI signals (post $>$ pre) on placebo and control sides. The rACC was uniquely activated during pain stimulation after placebo treatment on placebo sides (Fig. 2C). We also observed a negative correlation in bilateral rACC between each subject's fMRI signal change difference and the cor- 
responding pain rating difference. That is, the greater the placebo analgesia, the greater the activity in rACC. Note that the rACC activation in our study is located in the pregenual ACC (pACC) (Vogt, 2005), similar to (Petrovic et al., 2002; Zubieta et al., 2005) or slightly anterior to (Lieberman et al., 2004; Wager et al., 2004) other placebo analgesia studies. The pACC contains a high level of opioid receptors (Vogt et al., 1993); thus, our results support the view that endogenous opioids may be involved in expectancy enhanced acupuncture placebo analgesia.

Previous studies suggest that the ACC plays a pivotal role in pain perception and modulation (Buchel et al., 2002; Petrovic and Ingvar, 2002; Mohr et al., 2005), emotion perception and regulation (Drevets, 2000; Davidson et al., 2002; Shin et al., 2004, 2005), and their interaction (Vogt, 2005). In particular, the pACC, located in the affective subdivision of the ACC (Bush et al., 2000), is linked to arousal associated with emotional/motivational processing (Critchley, 2004). Activity in the pACC is associated with positive affective states such as happiness (Vogt, 2005). Kalisch et al. (2005) suggest that activation in medial prefrontal/pACC and anterolateral prefrontal cortex modulates anticipatory anxiety evoked by pain. In a recent study in irritable bowel syndrome patients, Vase and colleagues suggested that reduction in negative emotions may be important to placebo analgesia effect (Vase et al., 2005). Thus, we speculate that the pACC may be involved in placebo analgesia as a topdown modulator of negative emotional responses evoked by pain.

The prefrontal and parietal cortices are believed to be involved in the cognitive and attentional aspects of pain (Peyron et al., 2000). Previous studies suggest that DLPFC is important for continuous monitoring of the external world, maintenance of information in short-term memory, and governing efficient performance in the presence of interfering stimuli (Bunge et al., 2001; Fuster, 2001). Lorenz et al. (2003) suggest that DLPFC exerts active control on pain perception by modulating corticosubcortical and corticocortical pathways. In our study, activation in bilateral DLPFC was observed when comparing placebo $>$ control. A negative correlation between fMRI signal change and subjective ratings was also observed in bilateral DLPFC. Additional work is needed to determine the specific function of the DLPFC in placebo analgesia.

Recently, Pariente et al. (2005) investigated a different aspect of placebo acupuncture by comparing brain responses to RA, SN, and $\mathrm{OP}$ treatment in osteoarthritis patients. In contrast to the present study, their imaging data were collected during treatment, not during provocation of pre-existing pain in patients. They found that both RA and SN evoked greater activation than OP (no treatment effect expected) in the right rACC, DLPFC, and midbrain. The rACC activations in the two studies are located in different subdivision of the ACC. The rACC activation in our study was in pACC, whereas theirs was in the dorsal part of pMCC, which is primarily activated during acute somatic pain stimulation (Vogt, 2005).

In a previous study, Wager et al. (2004) found that after placebo cream treatment, fMRI signal decreased in pain sensitive regions such as thalamus, insula, and ACC. We did not find this result. Although similar, there are some methodological differences between the present study and that by Wager et al. One difference is the treatment: cream versus acupuncture. Another potentially important difference is that the study by Wager et al. applied placebo and control cream to different skin areas and compared only the post-treatment pain response on these different skin areas to detect placebo analgesia (their experiment 2). This method of using different skin areas has the advantage of avoiding possible confounds such as habituation to stimuli. In our study, we applied pain on the same skin areas before and after treatment and then compared the pretreatment and posttreatment differences between placebo and control sides to investigate the placebo analgesia effect. Our method has the advantage of avoiding differences in sensitivity between different skin areas on the arm.

In an attempt to see whether we could observe a similar result as the Wager group, we compared only the post-treatment pain 
Table 5. Brain areas activated during the comparison of fMRI signal change evoked by post-treatment high pain on placebo and control sides

\begin{tabular}{|c|c|c|c|c|}
\hline fMRI signal change & Area (Brodmann area) & Zscore & $\begin{array}{l}\text { Number of voxels } \\
\text { in cluster }\end{array}$ & Peak coordinate $(x, y, z)$ \\
\hline \multicolumn{5}{|l|}{ Within mask } \\
\hline \multirow[t]{5}{*}{$P>C$} & Right thalamus & 3.54 & 34 & $2,-14,8$ \\
\hline & Right anterior insula & 3.43 & 47 & $46,20,-4$ \\
\hline & Left SII $(2,40)$ & 3.28 & 83 & $-62,-26,24$ \\
\hline & Left thalamus & 3.21 & 33 & $-4,-12,8$ \\
\hline & Left pons & 3.12 & 29 & $-8,-32,-36$ \\
\hline$C>P$ & Nothing above threshold & & & \\
\hline \multicolumn{5}{|l|}{ Outside mask } \\
\hline \multirow[t]{11}{*}{$P>C$} & Right inferior frontal gyrus (47)/insula & 4.36 & 1717 & $56,28,2$ \\
\hline & Right inferior parietal cortex (40) & 4.28 & 766 & $56,-50,32$ \\
\hline & Posterior cingulate (31) & 4.22 & 801 & $8,-34,34$ \\
\hline & Bilateral rACC & 4.03 & 578 & $4,44,8$ \\
\hline & Right inferior parietal cortex (40) & 4 & 263 & $62,-30,50$ \\
\hline & Left inferior parietal cortex (40) & 3.94 & 574 & $56,-54,46$ \\
\hline & Left middle/inferior frontal gyrus $(10,46)$ & 3.60 & 91 & $-50,44,12$ \\
\hline & Right superior frontal gyrus (8) & 3.52 & 264 & $18,32,50$ \\
\hline & Right superior frontal gyrus (10) & 95 & 3.39 & $4,68,16$ \\
\hline & Left inferior frontal gyrus (44) & 23 & 3.05 & $-46,6,26$ \\
\hline & Left inferior frontal gyrus (47) & 50 & 3.04 & $-50,20,-6$ \\
\hline$C>P$ & Nothing above threshold & & & \\
\hline
\end{tabular}

The threshold is set to voxel-wise $p<0.005$ with 20 continuous voxels for predefined ROls and voxel-wise $p<0.001$ uncorrected with 20 contiguous voxels and cluster $p<0.05$ corrected for other regions. Peak coordinates refer to the MNI atlas. P, Placebo; C, control.

rating on the placebo and control sides instead of using the pretreatment and post-treatment differences. The results showed that there is a significant decrease in pain rating $(p<0.005)$ on the placebo side compared with the control side for high pain but not low pain. One possible reason for this discrepancy is that the pretreatment baseline rating for low pain on the placebo and control sides are slightly different (Table 3), and for high pain they are almost the same. Additional fMRI analysis of the posttreatment high pain level showed signal increases (placebo $>$ control; $p<0.005$ with 20 continuous voxels) in brain regions including bilateral medial thalamus, left SII and pons, and right AI within the mask (high pain $>$ low pain) and bilateral lateral prefrontal cortex, parietal cortex, and rACC outside the mask (Table 5). No activation was observed with the contrast of control $>$ placebo. This agrees with our initial analysis and differs from the study by Wager et al. (2004).

Previous studies indicate that placebo analgesia is a complicated phenomenon with different mechanisms at work under different conditions (Amanzio and Benedetti, 1999; Colloca and Benedetti, 2005). Reports suggest that as incoming sensory input becomes part of conscious awareness, the brain does not always directly reflect incoming signals from primary sensory neurons (Melzack and Katz, 1994; Petrovic and Ingvar, 2002). Instead, it undergoes extensive associative elaboration and modulation (Mesulam, 1998). Such corticosubcortical and corticocortical modulation by previous experience and expectation of result mediated by brain regions such as the DLPFC, pACC, and AI may also play an important role in the placebo effect. Placebo analgesia may be the result of a combination of components, the ratio of which varies depending on circumstance. Thus, we speculate that our study and study by Wager et al. (2004) measured different aspects or produced a different ratio of placebo analgesia components, for instance opioid and nonopioid components, of the placebo phenomena.

Left M1 is the only region that experienced greater activation in the control versus placebo condition. Wall (2000) proposes that pain is not simply a sensation but a need state like hunger or thirst involving cognitive, affective, and sensory components. As a need state, pain is associated with a requisite action, in this case preparing to withdraw from a noxious stimulus. We speculate the pain relief from the placebo effect may reduce this preparation and, thus, produce decreased activation in the motor region (M1) directing such movement.

In summary, we found placebo acupuncture treatment can produce a detectable placebo analgesic effect after expectancy manipulation. Brain regions including the right $\mathrm{AI}$, bilateral rACC, DLPFC, and parietal cortex may play an important role in this process. Different ratios of these components in different circumstances, like the modality of placebo treatment, could result in different brain activation patterns. Placebo analgesia may be configured through multiple brain pathways and mechanisms.

\section{References}

Amanzio M, Benedetti F (1999) Neuropharmacological dissection of placebo analgesia: expectation-activated opioid systems versus conditioning-activated specific subsystems. J Neurosci 19:484-494.

Beecher HK (1959) Reaction factors of the pain experience. In: Measurement of subjective responses: quantitative effects of drugs, Chap 9, pp 157-174. New York: Oxford UP.

Benedetti F, Arduino C, Amanzio M (1999) Somatotopic activation of opioid systems by target-directed expectations of analgesia. J Neurosci 19:3639-3648.

Buchel C, Bornhovd K, Quante M, Glauche V, Bromm B, Weiller C (2002) Dissociable neural responses related to pain intensity, stimulus intensity, and stimulus awareness within the anterior cingulate cortex: a parametric single-trial laser functional magnetic resonance imaging study. J Neurosci 22:970-976.

Bunge SA, Ochsner KN, Desmond JE, Glover GH, Gabrieli JD (2001) Prefrontal regions involved in keeping information in and out of mind. Brain 124:2074-2086.

Bush G, Luu P, Posner MI (2000) Cognitive and emotional influences in anterior cingulate cortex. Trends Cogn Sci 4:215-222.

Colloca L, Benedetti F (2005) Placebos and painkillers: is mind as real as matter? Nat Rev Neurosci 6:545-552.

Craig AD (2002) How do you feel? Interoception: the sense of the physiological condition of the body. Nat Rev Neurosci 3:655-666.

Craig AD (2003) A new view of pain as a homeostatic emotion. Trends Neurosci 26:303-307.

Craig AD, Chen K, Bandy D, Reiman EM (2000) Thermosensory activation of insular cortex. Nat Neurosci 3:184-190.

Critchley HD (2004) The human cortex responds to an interoceptive challenge. Proc Natl Acad Sci USA 101:6333-6334.

Critchley HD, Mathias CJ, Dolan RJ (2002) Fear conditioning in humans: the influence of awareness and autonomic arousal on functional neuroanatomy. Neuron 33:653-663.

Critchley HD, Wiens S, Rotshtein P, Ohman A, Dolan RJ (2004) Neural systems supporting interoceptive awareness. Nat Neurosci 7:189-195.

Davidson RJ, Lewis DA, Alloy LB, Amaral DG, Bush G, Cohen JD, Drevets WC, Farah MJ, Kagan J, McClelland JL, Nolen-Hoeksema S, Peterson BS (2002) Neural and behavioral substrates of mood and mood regulation. Biol Psychiatry 52:478-502.

Davis KD, Kwan CL, Crawley AP, Mikulis DJ (1998) Functional MRI study of thalamic and cortical activations evoked by cutaneous heat, cold and tactile stimuli. J Neurophysiol 80:1533-1546.

De Pascalis V, Chiaradia C, Carotenuto E (2002) The contribution of suggestibility and expectation to placebo analgesia phenomenon in an experimental setting. Pain 96:393-402. 
Drevets WC (2000) Neuroimaging studies of mood disorders. Biol Psychiatry $48: 813-829$.

Fuster JM (2001) The prefrontal cortex-an update: time is of the essence. Neuron 30:319-333.

Gracely RH, McGrath PA, Dubner R (1978a) Ratio scales of sensory and affective verbal pain descriptors. Pain 5:5-18.

Gracely RH, McGrath PA, Dubner R (1978b) Validity and sensitivity of ratio scales of sensory and affective verbal pain descriptors: manipulation of affect by diazepam. Pain 5:19-29.

Gracely RH, Dubner R, McGrath PA (1979) Narcotic analgesia: fentanyl reduces the intensity but not the unpleasantness of painful tooth pulp sensations. Science 203:1261-1263.

Gracely RH, Dubner R, Wolskee PJ, Deeter WR (1983) Placebo and naloxone can alter post-surgical pain by separate mechanisms. Nature 306:264-265.

Grevert P, Albert LH, Goldstain A (1983) Partial antagonism of placebo analgesia by naloxone. Pain 16:129-143.

Hoffman GA, Harrington A, Fields HL (2005) Pain and the placebo: what we have learned. Perspect Biol Med 48:248-265.

Kalisch R, Wiech K, Critchley HD, Seymour B, O’Doherty JP, Oakley DA, Allen P, Dolan RJ (2005) Anxiety reduction through detachment: subjective, physiological, and neural effects. J Cogn Neurosci 17:874-883.

Kaptchuk TJ (2002a) Acupuncture: theory, efficacy, and practice. Ann Intern Med 136:374-383.

Kaptchuk TJ (2002b) The placebo effect in alternative medicine: can the performance of a healing ritual have clinical significance? Ann Intern Med 136:817-825.

Kleinhenz J, Streitberger K, Windeler J, Gussbacher A, Mavridis G, Martin E (1999) Randomised clinical trial comparing the effects of acupuncture and a newly designed placebo needle in rotator cuff tendinitis. Pain 83:235-241.

Kong J, Fufa DT, Gerber AJ, Rosman IS, Vangel MG, Gracely RH, Gollub RL (2005a) Psychophysical outcomes from a randomized pilot study of manual, electro, and sham acupuncture treatment on experimentally induced thermal pain. J Pain 6:55-64.

Kong J, White N, Kwong KK, Vangel MG, Rosman IS, Gracely RH, Gollub RL (2005b) Using fMRI to dissociate sensory encoding from cognitive evaluation of heat pain intensity. Hum Brain Mapp, in press.

Kwan CL, Crawley AP, Milulis DJ, Davis KD (2000) An fMRI study of the anterior cingulate cortex and surrounding medial wall activations evoked by noxious cutaneous heat and cold stimuli. Pain 359-374.

Levine JD, Gordon NC, Fields HL (1978) The mechanism of placebo analgesia. Lancet 2:654-657.

Lieberman MD, Jarcho JM, Berman S, Naliboff BD, Suyenobu BY, Mandelkern M, Mayer EA (2004) The neural correlates of placebo effects: a disruption account. NeuroImage 22:447-455.

Lorenz J, Minoshima S, Casey KL (2003) Keeping pain out of mind: the role of the dorsolateral prefrontal cortex in pain modulation. Brain 126:1079-1091.

Melzack R, Katz J (1994) Pain measurement in persons in pain. In: Textbook of pain (Wall PD, Melzack R, eds), pp 337-351. Edinburgh: Churchill Livingstone.

Mesulam MM (1998) From sensation to cognition. Brain 121:1013-1052.

Mohr C, Binkofski F, Erdmann C, Buchel C, Helmchen C (2005) The anterior cingulate cortex contains distinct areas dissociating external from self-administered painful stimulation: a parametric fMRI study. Pain 114:347-357.
Montgomery GH, Kirsch I (1997) Classical conditioning and the placebo effect. Pain 72:107-113.

Pariente J, White P, Frackowiak RS, Lewith G (2005) Expectancy and belief modulate the neuronal substrates of pain treated by acupuncture. NeuroImage 25:1161-1167.

Petrovic P, Ingvar M (2002) Imaging cognitive modulation of pain processing. Pain 95:1-5.

Petrovic P, Kalso E, Petersson KM, Ingvar M (2002) Placebo and opioid analgesia-imaging a shared neuronal network. Science 295:1737-1740.

Peyron R, Garcia-Larrea L, Gregoire M, Costes N, Convers P, Lavenne F, Mauguiere F, Michel D, Laurent B (1999) Haemodynamic brain responses to acute pain in humans: sensory and attentional networks. Brain 122:1765-1779.

Peyron R, Laurent B, Garcia-Larrea L (2000) Functional imaging of brain responses to pain. A review and meta-analysis (2000). Neurophysiol Clin 30:263-288.

Price DD, Milling LS, Kirsch I, Duff A, Montgomery GH, Nicholls SS (1999) An analysis of factors that contribute to the magnitude of placebo analgesia in an experimental paradigm. Pain 83:147-156.

Roberts AH, Kewman DG, Mercier L, Hovell M (1993) The power of nonspecific effects in healing: implications for psychosocial and biological treatments. Clin Psychol Rev 12:375-391.

Shin LM, Orr SP, Carson MA, Rauch SL, Macklin ML, Lasko NB, Peters PM, Metzger LJ, Dougherty DD, Cannistraro PA, Alpert NM, Fischman AJ, Pitman RK (2004) Regional cerebral blood flow in the amygdala and medial prefrontal cortex during traumatic imagery in male and female Vietnam veterans with PTSD. Arch Gen Psychiatry 61:168-176.

Shin LM, Wright CI, Cannistraro PA, Wedig MM, McMullin K, Martis B, Macklin ML, Lasko NB, Cavanagh SR, Krangel TS, Orr SP, Pitman RK, Whalen PJ, Rauch SL (2005) A functional magnetic resonance imaging study of amygdala and medial prefrontal cortex responses to overtly presented fearful faces in posttraumatic stress disorder. Arch Gen Psychiatry 62:273-281.

Streitberger K, Kleinhenz J (1998) Introducing a placebo needle into acupuncture research. Lancet 352:364-365.

Vase L, Robinson ME, Verne GN, Price DD (2005) Increased placebo analgesia over time in irritable bowel syndrome (IBS) patients is associated with desire and expectation but not endogenous opioid mechanisms. Pain 115:338-347.

Vogt BA (2005) Pain and emotion interactions in subregions of the cingulate gyrus. Nat Rev Neurosci 6:533-544.

Vogt BA, Watanabe H, Grootoonk S, Jones AKP (1993) Topography of diprenorphine binding in human cingulate gyrus and adjacent cortex derived from coregistered PET and MR images. Hum Brain Mapp 3:1-12.

Voudouris NJ, Peck CL, Coleman G (1990) The role of conditioning and verbal expectancy in the placebo response. Pain 43:121-128.

Wager TD, Rilling JK, Smith EE, Sokolik A, Casey KL, Davidson RJ, Kosslyn SM, Rose RM, Cohen JD (2004) Placebo-induced changes in FMRI in the anticipation and experience of pain. Science 303:1162-1167.

Wall P (2000) Your Pain. In: Pain: the science of suffering, pp 141-157. New York: Columbia UP.

White P, Lewith G, Hopwood V, Prescott P (2003) The placebo needle, is it a valid and convincing placebo for use in acupuncture trials? A randomised, single-blind, cross-over pilot trial. Pain 106:401-409.

Zubieta JK, Bueller JA, Jackson LR, Scott DJ, Xu Y, Koeppe RA, Nichols TE, Stohler CS (2005) Placebo effects mediated by endogenous opioid activity on $\mu$-opioid receptors. J Neurosci 25:7754-7762. 